Aim of the study: Genistein, an isoflavonoid, plays roles in the inhibition of protein tyrosine kinase phosphorylation, induction of apoptosis, and cell differentiation in breast cancer. This study aims to induce cellular stress by exposing genistein to determine alterations of miRNA expression profiles in MCF-7 cells.

Material and methods: XTT assay and trypan blue dye exclusion assays were performed to examine the cytotoxic effects of genistein treatment. Expressions of miRNAs were quantified us ing Real-Time Online RT-PCR.

Results: The $\mathrm{IC}_{50}$ dose of genistein was $175 \mu \mathrm{M}$ in MCF-7 cell, line and the cytotoxic effect of genistein was detected after 48 hours. miR-23b was found to be up-regulated 56.69 fold following the treatment of genistein. It was found that miR-23b was upregulated for MCF-7 breast cancer cells after genistein treatment.

Conclusions: Up-regulated ex-expression of miR-23b might be a putative biomarker for use in the therapy of breast cancer patients. miR-23b up-regulation might be important in terms of response to genistein.

Key words: breast cancer, genistein, miRNA, MCF-7.

Contemp Oncol (Pozn) 2015; 19 (1): 32-35 DOI: $10.5114 /$ wo.2014.4412

\section{Genistein-induced mir-23b expression inhibits the growth of breast cancer cells}

\author{
Cigir Biray Avci ${ }^{1}$, Sunde Yilmaz Susluer ${ }^{1}$, Hasan Onur Caglar ${ }^{2}$, Tugce Balci ${ }^{1}$, \\ Duygu Aygunes ${ }^{1}$, Yavuz Dodurga ${ }^{3}$, Cumhur Gunduz ${ }^{1}$
}

${ }^{1}$ Department of Medical Biology, Medical Faculty, Ege University, Bornova, Izmir, Turkey ${ }^{2}$ Department of Stem Cell, Health Science Institute, Ege University, Bornova, Izmir, Turkey

${ }^{3}$ Department of Medical Biology, School of Medicine, Pamukkale University, Denizli, Turkey

\section{Introduction}

Breast cancer is the most common cancer type among women. After respiratory cancers, including lung and respiratory tract cancers, breast cancer is the second leading cause of death among women [1]. Incidence and mortality rates of breast cancer may differ depending on the age, ethnicity, and socioeconomic status of patients [2]. The presence or absence of oestrogen receptor (ER), progesterone receptor (PR), and HER2/Neu receptor determine the sub-molecular classification and progression of breast cancer [3]. miRNAs are non-coding small RNA molecules (17-24 nucleotide) that repress mRNA transcription when they bind to their target region (3' untranslated region). Reregulation of expression profiles of miRNAs has a putative role in cancer development [4]. Decreasing expression of miRNA that suppress mRNA of oncogene, or increasing of expression of miRNA that suppress mRNA of tumour suppressor gene, can trigger the cancer process $[4,5]$. It has been demonstrated that oncomirs such as miR-21, miR-27a, miR-155, and miR-145 exhibit different expression patterns between breast cancer cells and non-cancerous breast cells [6-8]. In addition to this difference, altered miRNA expression profile is detected differently among molecular sub-types of breast cancer [9]. Abnormal miRNA expression pattern can induce angiogenesis and metastasis in breast cancer tumours [10]. miRNA dysregulation in breast cancer is also associated with poor survival and poor therapeutic outcome [11, 12].

Genistein, an isoflavonoid, is a prime anti-cancer component of soybean, and it plays roles in the inhibition of protein tyrosine kinase phosphorylation, induction of apoptosis, and cell differentiation in breast cancer [13]. The interaction between genistein and oestrogen receptor signalling pathway in breast cancer has been well characterised. Genistein induces oestrogen-dependent cell growth and up-regulation of ER expression, thus it has a potential impact for hormone therapy [14, 15]. Genistein-mediated ER $\alpha$ expression is associated with histone modification changes and genistein re-sensitises ER $\alpha$-negative breast cancer cells to tamoxifen [16]. Although it has been known that genistein affects chemotherapy agent efficiency and apoptosis, the effect of genistein on miRNA profiles is still unknown for breast cancer. This study aims to induce cellular stress by exposing genistein to determine the $I C_{50}$ doses of treatment conditions in MCF-7 cells. This study also aims to evaluate the single effect in terms of miRNA expression levels.

\section{Material and methods}

Tumour cell line

Breast cancer cell line (MCF-7), which was purchased from ATCC, was used as a breast cancer model. 


\section{Cell culture}

Breast cancer cell line (MCF-7) was cultured in RPMI1640 medium supplemented with $100 \mathrm{IU} / \mathrm{ml}$ penicillin, $10 \mathrm{mg} / \mathrm{ml}$ streptomycin, $1 \%$ L-glutamine, and 10\% heat-inactivated foetal bovine serum, at $37^{\circ} \mathrm{C}$ in a humidified 95\% air 5\% $\mathrm{CO}_{2}$ atmosphere.

\section{Treatment of genistein and cytotoxicity assay}

MCF-7 cells were incubated at a density of $2 \times 10^{5}$ cells/ $\mathrm{ml}$ of medium using 96 -well plates for 24,48 , and 72 hours. Studied concentrations of genistein were $75 \mu \mathrm{M}$, $100 \mu \mathrm{M}, 125 \mu \mathrm{M}, 150 \mu \mathrm{M}, 175 \mu \mathrm{M}$, and $200 \mu \mathrm{M}$. XTT assay and trypan blue dye exclusion assays were performed to examine the cytotoxic effect of $I C_{50}$ dose of genistein in the MCF-7 cell line. Formazan formation was quantified spectrophotometrically at $450 \mathrm{nM}$ (reference wavelength 620 nM) with a microplate reader. Viability was calculated using the background-corrected absorbance. Cells without any treatment were taken as a control group.

\section{Isolation of miRNA}

miRNA was isolated from cells exposed to $I C_{50}$ dose of genistein and the control group. Isolation of miRNA and CDNA synthesis was performed using RT $^{2}$ qPCR-Grade miRNA Isolation Kit and $\mathrm{RT}^{2}$ first Strand Kit, respectively, according to the manufacturers' instructions.
Relative quantification of miRNAs

Relative quantitation of 88 microRNAs (Table 1) was measured by using real-time online RT-PCR (LightCycler 480). SNORD48, SNORD47, SNORD44, U6 were used as human endogenous controls. Alterations in the miRNAs expressions of genistein were compared to the control group. Data analysis was evaluated by $\Delta \Delta C T$ method, "Light Cycler ${ }^{\circledR} 480$ Quantification Software" program, and statistical analysis was evaluated with web-based RT $^{2}$ Profiler PCR Array Data Analysis.

\section{Results}

\section{Cytotoxic effect of genistein on MCF-7 cells}

Cells were incubated at a density of $2 \times 10^{5}$ cells $/ \mathrm{ml}$ of medium using 96-well plates for 24,48 , and 72 hours. Studied concentrations of genistein were $75 \mu \mathrm{M}, 100 \mu \mathrm{M}$, $125 \mu \mathrm{M}, 150 \mu \mathrm{M}, 175 \mu \mathrm{M}$, and $200 \mu \mathrm{M}$ (Fig. 1). Untreated MCF-7 cells were considered as a control group. The $\mathrm{IC}_{50}$ dose of genistein was $175 \mu \mathrm{M}$ and the cytotoxic effect of genistein was detected after 48 hours.

miR-23b is up-regulated miRNA by genistein in MCF-7 cell line

Alterations in the expressions of miRNAs were compared with genistein untreated MCF-7 cells. miRNA expression was detected 48 hours after genistein treatment. SNORD44, SNORD47, SNORD48, and U6 genes were

Table 1. Target and housekeeping miRNAs that were analysed for the genistein group. Expression analyses for 88 target miRNAs were performed by real-time PCR. Table 1 describes the sequences of miRNAs

\begin{tabular}{|c|c|c|c|c|c|}
\hline miRNA & Sequence & miRNA & Sequence & miRNA & Sequence \\
\hline hsa-miR-142-5p & CAUAAAGUAGAAAGCACUACU & hsa-miR-191 & CAACGGAAUCCCAAAAGCAGCUG & hsa-miR-320 & AAAAGCUGGGUUGAGAGGGCGA \\
\hline hsa-miR-16 & UAGCAGCACGUAAAUAUUGGCG & hsa-miR-17 & CAAAGUGCUUACAGUGCAGGUAG & hsa-miR-374a & UUAUAAUACAACCUGAUAAGUG \\
\hline hsa-miR-142-3p & UGUAGUGUUUCCUACUUUAUGGA & hsa-miR-130a & CAGUGCAAUGUUAAAAGGGCAU & hsa-let-7e & UGAGGUAGGAGGUUGUAUAGUU \\
\hline hsa-miR-21 & UAGCUUAUCAGACUGAUGUUGA & hsa-miR-20a & UAAAGUGCUUAUAGUGCAGGUAG & hsa-miR-151-5p & UCGAGGAGCUCACAGUCUAGU \\
\hline hsa-miR-15a & UAGCAGCACAUAAUGGUUUGUG & hsa-miR-27b & UUCACAGUGGCUAAGUUCUGC & hsa-miR-374b & AUAUAAUACAACCUGCUAAGUG \\
\hline hsa-miR-29b & UAGCACCAUUUGAAAUCAGUGUU & hsa-miR-26b & UUCAAGUAAUUCAGGAUAGGU & hsa-miR-196b & UAGGUAGUUUCCUGUUGUUGGG \\
\hline hsa-let-7a & UGAGGUAGUAGGUUGUAUAGUU & hsa-miR-146a & UGAGAACUGAAUUCCAUGGGUU & hsa-miR-140-3p & UACCACAGGGUAGAACCACGG \\
\hline hsa-miR-126 & UCGUACCGUGAGUAAUAAUGCG & hsa-miR-200c & UAAUACUGCCGGGUAAUGAUGGA & hsa-miR-100 & AACCCGUAGAUCCGAACUUGUG \\
\hline hsa-miR-143 & UGAGAUGAAGCACUGUAGCUC & hsa-miR-99a & AACCCGUAGAUCCGAUCUUGUG & hsa-miR-103 & AGCAGCAUUGUACAGGGCUAUGA \\
\hline hsa-let-7b & UGAGGUAGUAGGUUGUGUGGUU & hsa-miR-19a & UGUGCAAAUCUAUGCAAAACUGA & hsa-miR-96 & UUUGGCACUAGCACAUUUUUGCU \\
\hline hsa-miR-27a & UUCACAGUGGCUAAGUUCCGC & hsa-miR-23a & AUCACAUUGCCAGGGAUUUCC & hsa-miR-302b & UAAGUGCUUCCAUGUUUUAGUAG \\
\hline hsa-let-7f & UGAGGUAGUAGAUUGUAUAGUU & hsa-miR-30a & UGUAAACAUCCUCGACUGGAAG & hsa-miR-194 & UGUAACAGCAACUCCAUGUGGA \\
\hline hsa-miR-9 & UCUUUGGUUAUCUAGCUGUAUGA & hsa-let-7i & UGAGGUAGUAGUUUGUGCUGUU & hsa-miR-125a-5p & UCCCUGAGACCCUUUAACCUGUGA \\
\hline hsa-miR-26a & UUCAAGUAAUCCAGGAUAGGCU & hsa-miR-93 & CAAAGUGCUGUUCGUGCAGGUAG & hsa-miR-423-5p & UGAGGGGCAGAGAGCGAGACUUU \\
\hline hsa-miR-24 & UGGCUCAGUUCAGCAGGAACAG & hsa-let-7c & UGAGGUAGUAGGUUGUAUGGUU & hsa-miR-376c & AACAUAGAGGAAAUUCCACGU \\
\hline hsa-miR-30e & UGUAAACAUCCUUGACUGGAAG & hsa-miR-106b & UAAAGUGCUGACAGUGCAGAU & hsa-miR-195 & UAGCAGCACAGAAAUAUUGGC \\
\hline hsa-miR-181a & AACAUUCAACGCUGUCGGUGAGU & hsa-miR-101 & UACAGUACUGUGAUAACUGAA & hsa-miR-222 & AGCUACAUCUGGCUACUGGGU \\
\hline hsa-miR-29a & UAGCACCAUCUGAAAUCGGUUA & hsa-let-7g & UGAGGUAGUAGUUUGUACAGUU & hsa-miR-28-3p & CACUAGAUUGUGAGCUCCUGGA \\
\hline hsa-miR-124 & UAAGGCACGCGGUGAAUGCC & hsa-miR-425 & AAUGACACGAUCACUCCCGUUGA & hsa-miR-128a & UCACAGUGAACCGGUCUCUUU \\
\hline hsa-miR-144 & UACAGUAUAGAUGAUGUACU & hsa-miR-15b & UAGCAGCACAUCAUGGUUUAACA & hsa-miR-302c & UAAGUGCUUCCAUGUUUCAGUGG \\
\hline hsa-miR-30d & UGUAAACAUCCCCGACUGGAAG & hsa-miR-28-5p & AAGGAGCUCACAGUCUAUUGAG & hsa-miR-423-3p & AGCUCGGUCUGAGGCCCCUCAGU \\
\hline hsa-miR-19b & UGUGCAAAUCCAUGCAAAACUGA & hsa-miR-18a & UAAGGUGCAUCUAGUGCAGAUAG & hsa-miR-185 & UGGAGAGAAAGGCAGUUCCUGA \\
\hline hsa-miR-22 & AAGCUGCCAGUUGAAGAACUGU & hsa-miR-25 & CAUUGCACUUGUCUCGGUCUGA & hsa-miR-30b & UGUAAACAUCCUACACUCAGCU \\
\hline hsa-miR-122 & UGGAGUGUGACAAUGGUGUUUG & hsa-miR-23b & AUCACAUUGCCAGGGAUUACC & hsa-miR-210 & CUGUGCGUGUGACAGCGGCUGA \\
\hline hsa-miR-150 & UCUCCCAACCCUUGUACCAGUG & hsa-miR-302a & UAAGUGCUUCCAUGUUUUGGUGA & SNORD48 & TAACTCTGAGTGTGTCGCTGA \\
\hline hsa-miR-32 & UAUUGCACAUUACUAAGUUGCA & hsa-miR-186 & CAAAGAAUUCUCCUUUUGGGCU & SNORD47 & CCGTTCCATTTTGATTCTGAG \\
\hline hsa-miR-155 & UUAAUGCUAAUCGUGAUAGGGGU & hsa-miR-29c & UAGCACCAUUUGAAAUCGGUUA & SNORD44 & GGTCTTAATTAGCTCTAACTGAC \\
\hline hsa-miR-140-5p & CAGUGGUUUUACCCUAUGGUAG & hsa-miR-7 & UGGAAGACUAGUGAUUUUGUUGU & U6 & ATTGGAACGATACAGAGAAGATTAG \\
\hline hsa-miR-125b & UCCCUGAGACCCUAACUUGUGA & hsa-let-7d & AGAGGUAGUAGGUUGCAUAGUU & miRTC & ACACTAAGTACGTCGTATTAC \\
\hline hsa-miR-141 & UAACACUGUCUGGUAAAGAUGG & hsa-miR-30c & UGUAAACAUCCUACACUCUCAGC & miRTC & ACACTAAGTACGTCGTATTAC \\
\hline hsa-miR-92a & UAUUGCACUUGUCCCGGCCUGU & hsa-miR-181b & AACAUUCAUUGCUGUCGGUGGGU & & \\
\hline hsa-miR-424 & CAGCAGCAAUUCAUGUUUUGAA & hsa-miR-223 & UGUCAGUUUGUCAAAUACCCCA & & \\
\hline
\end{tabular}




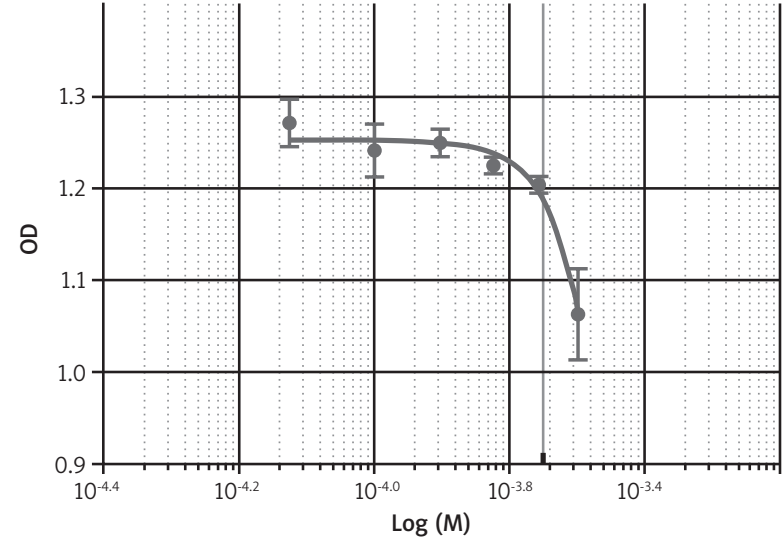

Fig. 1. Dose-dependent cytotoxicity of genistein. MCF-7 cells were treated with various concentrations of genistein. The studied concentrations of genistein were $75 \mu \mathrm{M}, 100 \mu \mathrm{M}, 125 \mu \mathrm{M}, 150 \mu \mathrm{M}, 175 \mu \mathrm{M}$, and $200 \mu \mathrm{M}$. The IC50 dose of genistein was $175 \mu \mathrm{M}$

used for housekeeping miRNAs as the endogenous normalisation factor to define miRNA expression profiles of 88 miRNAs. miR-23b was found to be up-regulated 56.69 fold in the treatment of genistein compared to the control group of genistein untreated cells (Fig. 2).

\section{Discussion}

Several studies have reported that genistein, which is an isoflavonoid and is a prime anti-cancer component
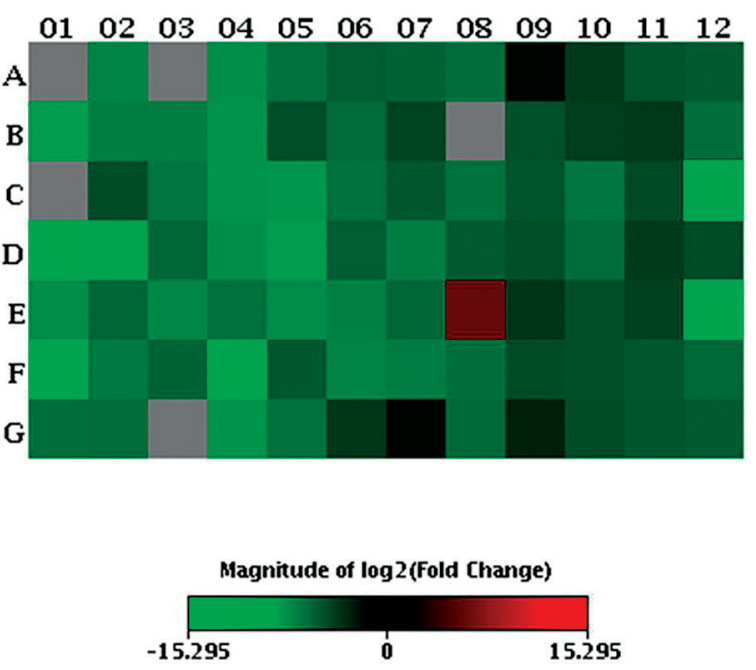

of soybean, can affect miRNA expression levels [17-20]. miR-151, which has an oncogenic effect, is up-regulated in prostate cancer cell lines (PCa), and genistein treatment down-regulates the relative expression of miR-151 in PCa [21]. It is known that genistein induces expression of miR574-3p, which has a tumour suppressor role, and this induction inhibits cell proliferation, migration, and invasion in vitro and in vivo for prostate cancers [22]. Zaman et al. showed that genistein decreases the expression of miR23b-3p in A-498 renal cancer cell line [23]. Furthermore, suppression of miR-23b-3p increases the number of total apoptotic cells and decreases cell invasion [23]. Although it is known that genistein affects chemotherapy agent efficiency and apoptosis, the effect of genistein on miRNA profiles is still unknown for breast cancer. In this study, it was found that treatment condition, which was genistein, affected miRNA expressions in MCF-7 breast cancer cell line. The cytotoxic effects of the defined group were examined independently. Cells in the genistein group were treated with an $\mathrm{IC}_{50}$ dose of genistein for three days. The cytotoxic effect of treatment group was observed after 48 hours. After the $I C_{50}$ dose of genistein was determined, miRNA qPCR array method was performed to detect regulation of miRNAs expressions in MCF-7 cell line. In this study, it was found that expression of miR-23b was up-regulated in the genistein treatment group. Majid et al. clearly showed that miR-23b is a methylation-silenced tumour suppressor in prostate cancer, and a high expression level of miR-23b is associated with higher survival rates in prostate cancer patients [24]. Stabile ectopic expression of miR-23b in HCT-116 colon carcinoma cell line reduces migration, invasion, and resistance to anoikis [25]. In vivo tumour models, which are generated from miR-23b-expressing HCT 116 cells, show that miR-23b-expressing tumours are encapsulated, non-invasive, and have low growth rate [25]. miR$23 \mathrm{~b}$ regulates colony morphology and increases epithelial characteristics in MCF-7 cells. It is observed that miR-23b enhances focal adhesion connections and provides less lamellipodia structure after transfection in MDA-MB-231 breast cancer cells [26]. miR-23b regulates cytoskeletal reorganization and reduces cell motility and invasion via the PAK2 gene, which is a target for miR-23b in MCF-7 and MDA-MB-231 cells [26]. Furthermore, inhibition of miR-23b increases cell migration and metastasis for in vivo breast cancer models [26]. Because it is known that miR-23b has

\begin{tabular}{|c|c|c|c|c|c|c|c|c|c|c|c|c|}
\hline \multirow{3}{*}{ A } & $\overline{1}$ & 2 & 3 & 4 & 5 & 6 & 7 & 8 & 9 & 10 & 11 & 12 \\
\hline & miR-142-5p & miR-16 & miR-142-3p & miR-21 & miR-15a & miR-29b & let-7a & miR-126 & miR-143 & let-7b & miR-27a & let-7f \\
\hline & $-62,47$ & $-160,34$ & $-62,47$ & $-228,33$ & $-88,95$ & $-49,69$ & $-58,28$ & $-82,42$ & $-4,13$ & $-16,39$ & $-40,09$ & $-47,34$ \\
\hline \multirow[t]{2}{*}{ B } & miR-9 & miR-26a & miR-24 & miR-30e & miR-181a & miR-29a & miR-124 & miR-144 & miR-30d & miR-19b & miR-22 & miR-122 \\
\hline & $-360,79$ & $-132,06$ & $-131,14$ & $-267,8$ & $-33,47$ & $-76,37$ & $-24,17$ & $-62,47$ & $-34,9$ & $-19,49$ & $-17,57$ & -83 \\
\hline \multirow[t]{2}{*}{ c } & miR-150 & miR-32 & miR-155 & miR-140-5p & miR-125b & miR-141 & miR-92a & miR-424 & miR-191 & miR-17 & miR-130a & miR-20a \\
\hline & $-62,47$ & $-30,38$ & $-105,05$ & $-273,42$ & $-279,17$ & $-92,73$ & $-41,5$ & $-86,52$ & $-40,36$ & $-106,52$ & $-28,74$ & $-40202,57$ \\
\hline \multirow[t]{2}{*}{ D } & miR-27b & miR-26b & miR-146a & miR-200c & miR-99a & miR-19a & miR-23a & miR-30a & let-7i & miR-93 & let-7c & miR-106b \\
\hline & $-1108,97$ & $-489,44$ & $-65,57$ & $-217,52$ & $-373,51$ & $-50,39$ & $-137,66$ & $-47,34$ & $-33,94$ & $-73,26$ & $-17,81$ & $-31,23$ \\
\hline \multirow[t]{2}{*}{ E } & miR-101 & let-7g & miR-425 & miR-15b & miR-28-5p & miR-18a & miR-25 & miR-23b & miR-302a & miR-186 & miR-29c & miR-7 \\
\hline & $-217,52$ & $-62,03$ & $-168,31$ & $-92,73$ & $-197,4$ & $-150,64$ & $-65,12$ & 56,69 & $-14,07$ & $-31,89$ & $-21,63$ & $-6912,54$ \\
\hline \multirow[t]{2}{*}{ F } & let-7d & miR-30c & miR-181b & miR-223 & miR-320a & miR-374a & let-7e & miR-151-5p & miR-374b & miR-196b & miR-140-3p & miR-100 \\
\hline & $-590,18$ & $-118,19$ & $-59,1$ & $-716,59$ & $-42,67$ & $-153,81$ & $-123,21$ & $-85,33$ & $-29,96$ & $-32,56$ & $-42,08$ & $-69,31$ \\
\hline \multirow[t]{2}{*}{ G } & miR-103 & miR-96 & miR-302b & miR-194 & miR-125a-5p & miR-423-5p & miR-376c & miR-195 & miR-222 & miR-28-3p & miR-128 & miR-302c \\
\hline & $-80,17$ & $-75,85$ & $-62,47$ & $-264,11$ & $-92,09$ & $-15,94$ & $-4,18$ & $-71,26$ & $-7,75$ & $-30,38$ & $-38,72$ & $-47,34$ \\
\hline
\end{tabular}

Fig. 2. miRNA expression profiles after treatment. For the genistein group, miR-23b was up-regulated 56.69 fold after treatment. miRNA expression visualization about log2 (Fold Change) associated with genistein, compared with control 
a tumour suppressor role for metastasis of breast cancer cells, miR-23b up-regulation might be important in terms of response to genistein.

In conclusion, up-regulated expression of miR-23b might be a putative biomarker for use in the therapy of breast cancer patients.

The authors declare no conflict of interest.

This study was supported by Ege University Medical Faculty Research Project Subcommittee (Grant number:2008/ TIP/021).

\section{References}

1. Siegel R, Ward E, Brawley O, Jemal A. Cancer statistics, 2011: the impact of eliminating socioeconomic and racial disparities on premature cancer deaths. CA Cancer J Clin 2011; 61: 212-36.

2. DeSantis C, Siegel R, Bandi P, Jemal A. Breast cancer statistics, 2011. CA Cancer J Clin 2011; 61: 409-18.

3. Sorlie T, Perou CM, Tibshirani R, et al. Gene expression patterns of breast carcinomas distinguish tumor subclasses with clinical implications. Proc Natl Acad Sci U S A 2001; 98: 10869-74.

4. Hammond SM. RNAi, microRNAs, and human disease. Cancer Chemother Pharmacol 2006; 58 Suppl 1: s63-8.

5. Iorio MV, Casalini P, Piovan C, Braccioli L, Tagliabue E. Breast cancer and microRNAs: therapeutic impact. Breast 2011; 20 Suppl 3: s63-70.

6. Iorio MV, Ferracin M, Liu CG, et al. MicroRNA gene expression de regulation in human breast cancer. Cancer Res 2005; 65: 7065-70.

7. Guttilla IK, White BA. Coordinate regulation of FOXO1 by miR-27a, miR-96, and miR-182 in breast cancer cells. J Biol Chem 2009; 284: 23204-16.

8. Li L, Xiao B, Tong H, Xie F, Zhang Z, Xiao GG. Regulation of breast cancer tumorigenesis and metastasis by miRNAs. Expert Rev Proteomics 2012; 9: 615-25.

9. Lowery AJ, Miller N, Devaney A, et al. MicroRNA signatures predict oestrogen receptor, progesterone receptor and HER2/neu receptor status in breast cancer. Breast Cancer Res 2009; 11: R27.

10. Harquail J, Benzina S, Robichaud GA. MicroRNAs and breast cance malignancy: an overview of miRNA-regulated cancer processes leading to metastasis. Cancer Biomark 2012; 11: 269-80.

11. Lyng MB, Laenkholm AV, Sokilde R, Gravgaard KH, Litman T, Dit zel HJ. Global microRNA expression profiling of high-risk ER+ breast cancers from patients receiving adjuvant tamoxifen mono-therapy: a DBCG study. PLoS One 2012; 7: e36170.

12. Rothe F, Ignatiadis M, Chaboteaux C, et al. Global microRNA expression profiling identifies MiR-210 associated with tumor proliferation, invasion and poor clinical outcome in breast cancer. PLoS One 2011; 6: e20980.

13. Orlando L, Schiavone P, Cinieri S. Genistein: the future of prevention and treatment of breast cancer? Cancer Biol Ther 2011; 11: 918-20.

14. Beck V, Unterrieder E, Krenn L, Kubelka W, Jungbauer A. Comparison of hormonal activity (estrogen, androgen and progestin) of standardized plant extracts for large scale use in hormone replacement therapy. J Steroid Biochem Mol Biol 2003; 84: 259-68.

15. van Duursen MB, Nijmeijer SM, de Morree ES, de Jong PC, van den Berg M. Genistein induces breast cancer-associated aromatase and stimulates estrogen-dependent tumor cell growth in in vitro breast cancer model. Toxicology 2011; 289: 67-73.

16. Li Y, Meeran SM, Patel SN, Chen H, Hardy TM, Tollefsbol TO. Epigenetic reactivation of estrogen receptor-alpha (ERalpha) by genistein enhances hormonal therapy sensitivity in ERalpha-negative breast cancer. Mol Cancer 2013; 12: 9 .

17. Parker LP, Taylor DD, Kesterson J, Metzinger DS, Gercel-Taylor C. Modulation of microRNA associated with ovarian cancer cells by genistein. Eur J Gynaecol Oncol 2009; 30: 616-21.
18. Li Y, VandenBoom TG, 2nd, Kong D, Wang Z, Ali S, Philip PA, Sarkar FH. Up-regulation of miR-200 and let-7 by natural agents leads to the reversal of epithelial-to-mesenchymal transition in gemcitabine-resistant pancreatic cancer cells. Cancer Res 2009; 69: 6704-12

19. Li Y, Vandenboom TG, 2nd, Wang Z, Kong D, Ali S, Philip PA, Sarkar FH. miR-146a suppresses invasion of pancreatic cancer cells. Cancer Res 2010; 70: 1486-95.

20. Sun Q, Cong R, Yan H, et al. Genistein inhibits growth of human uveal melanoma cells and affects microRNA-27a and target gene expression. Oncol Rep 2009; 22: 563-7.

21. Chiyomaru T, Yamamura S, Zaman MS, et al. Genistein suppresses prostate cancer growth through inhibition of oncogenic micro RNA-151. PLoS One 2012; 7: e43812.

22. Chiyomaru T, Yamamura S, Fukuhara S, et al. Genistein up-regulates tumor suppressor microRNA-574-3p in prostate cancer. PLoS One 2013; 8: e58929.

23. Zaman MS, Thamminana S, Shahryari V, et al. Inhibition of PTEN gene expression by oncogenic miR-23b-3p in renal cancer. PLoS One 2012; 7: e50203.

24. Majid S, Dar AA, Saini S, et al. miR-23b represses proto-oncogene Src kinase and functions as methylation-silenced tumor suppressor with diagnostic and prognostic significance in prostate cancer. Cancer Res 2012; 72: 6435-46.

25. Zhang H, Hao Y, Yang J, Zhou Y, Li J, Yin S, Sun C, Ma M, Huang Y, Xi JJ. Genome-wide functional screening of miR-23b as a pleiotropic modulator suppressing cancer metastasis. Nat Commun 2011; 2: 554.

26. Pellegrino L, Stebbing J, Braga VM, et al. miR-23b regulates cytoskeletal remodeling, motility and metastasis by directly targeting multiple transcripts. Nucleic Acids Res 2013; 41: 5400-12.

\section{Address for correspondence}

Assistant Prof. Dr. Yavuz Dodurga, PhD

Department of Medical Biology

Pamukkale University

Denizli, Turkey

tel. +902582962534

e-mail: yavuzdodurga@gmail.com

Submitted: 31.03 .2014

Accepted: $\quad 30.06 .2014$ 